\title{
Evaluation of effect of preoperative chemotherapy on Wilms' tumor histopathology
}

\section{Taskinen, Seppo}

2018-08

Taskinen , S , Lohi , J , Koskenvuo , M \& Taskinen , M 2018 , ' Evaluation of effect of preoperative chemotherapy on Wilms' tumor histopathology ' , Journal of Pediatric Surgery , vol. 53 , no. 8 , pp. 1611-1614 . https://doi.org/10.1016/j.jpedsurg.2017.10.002

http://hdl.handle.net/10138/304164

https://doi.org/10.1016/j.jpedsurg.2017.10.002

publishedVersion

Downloaded from Helda, University of Helsinki institutional repository.

This is an electronic reprint of the original article.

This reprint may differ from the original in pagination and typographic detail.

Please cite the original version. 
Oncology

\title{
Evaluation of effect of preoperative chemotherapy on Wilms' tumor histopathology
}

\author{
Seppo Taskinen ${ }^{\mathrm{a}, *}$, Jouko Lohi ${ }^{\mathrm{b}}$, Minna Koskenvuo ${ }^{\mathrm{c}}$, Mervi Taskinen ${ }^{\mathrm{c}}$ \\ a Department of Pediatric Surgery, Children's Hospital, Helsinki University Hospital, Helsinki, Finland \\ b Department of Pathology, Helsinki University Hospital, Helsinki, Finland \\ c Division of Pediatric Hematology/Oncology and Stem Cell Transplantation, Children's Hospital, Helsinki University Hospital, Helsinki, Finland
}

\section{A R T I C L E I N F O}

\section{Article history:}

Received 12 June 2017

Received in revised form 26 September 2017

Accepted 1 October 2017

\section{Key words:}

Wilms tumor

Pathology

Kidney

Children

Chemotherapy

\begin{abstract}
A B S T R A C T
Purpose: To evaluate usefulness of cutting needle biopsy (CNB) to recognize pediatric renal tumors and to predict the evolution of histology during preoperative chemotherapy of Wilms tumors.

Methods: Ninety pediatric patients were operated for renal tumors at our institution in 1988-2015. We included all 64 patients who had undergone CNB at diagnosis and whose CNB and nephrectomy samples were available for re-evaluation.

Results: The CNB was diagnostic in all 59 Wilms tumors but only in two out of five non-Wilms tumors. Anaplasia was missed by $\mathrm{CNB}$ in one of three with diffuse anaplasia in nephrectomy specimens. In Wilms tumors the proportions of the blastemal, stromal and epithelial components were 55\% (IQR 25-85), 28\% (IQR 10-58) and $2 \%$ (IQR $0-10$ ) in CNB samples and 5\% (IQR 0-64), 15\% (IQR 0-50) and 15\% (IQR 0-44) in the nephrectomy specimens ( $\mathrm{p}$-values $0.002,0.599$ and 0.005 respectively). The degree of tumor necrosis was in median $80 \%$ (IQR 21-97), after preoperative chemotherapy. The degree of tumor necrosis after chemotherapy had a positive correlation with the proportion of blastemal component $(p=0.008)$ and a negative correlation with proportion of epithelial component in pre-chemotherapy CNB samples $(p<0.001)$.

Conclusions: Wilms tumors are usually recognizable unlike non-Wilms tumors in CNB at diagnosis. In Wilms tumors, high blastemal cell content is associated with significant tumor necrosis during pre-operative chemotherapy. Our results do not support routine use of CNB in diagnosis of renal tumors.

Type of study: Retrospective review.

Level of evidence: Level III.
\end{abstract}

(c) 2017 Elsevier Inc. All rights reserved.
Wilms' tumor is treated primarily using strategies created by the Wilms' tumor study (NWTSG), and its successor Children's Oncology Group (COG) Renal Tumor Committee or by International Society of Pediatric Oncology (SIOP) Renal Tumor Committee [1]. North-American NWTS/COG protocols are based on primary surgery followed by chemotherapy. This approach allows an accurate and comprehensive evaluation of tumor histology. The SIOP protocol is used mainly in Europe and an essential part of it has been preoperative chemotherapy. During

\footnotetext{
H. An abstract has been presented in the annual congress of European Society for Pediatric Urology 2017.

* Corresponding author at: Children's Hospital, Helsinki University Hospital, Stenbäckinkatu 11, 00290 Helsinki, Finland. Tel.: +358 504272542; fax: + 3589 47175314.

E-mail address: seppo.taskinen@hus.fi (S. Taskinen).
}

preoperative chemotherapy the tumor size shrinks and the operation becomes easier with a decreased risk for tumor rupture [2,3]. However, the preoperative chemotherapy may be directed to the wrong type of tumor, if histological verification is lacking, and accurate histological classification may occasionally be impossible from the nephrectomy specimen because of tumor necrosis. The use of cutting needle biopsy (CNB) was presented previously in 1991 in studies from Helsinki, London and Toronto, followed later by Sweden [4-7]. In general, positive experience has been reported from UK from the use of cutting needle biopsies: $12 \%$ of the patients who turned out to have non-Wilms' tumor avoided inappropriate chemotherapy [8]. The drawback of this approach is that the primary CNB represents small foci of the tumor. In addition, the diagnosis of anaplasia has been particularly challenging by CNB in prior reports [8].

Diagnostic biopsy and preoperative chemotherapy have been part of our protocol in children with a renal tumor since the initial report in 
Table 1

The chemotherapy administered pre-operative for all the children. The chemotherapy protocols used were mainly based on SIOP and NWTS protocols.

\begin{tabular}{lll}
\hline Preoperative chemotherapy $(\mathrm{n}=59)$ & Median, range & $\%$ of Children \\
\hline Dactinomycin $\mathrm{mg} / \mathrm{kg}$ & $0.17,0.06-0.29$ & $100 \%$ \\
Vincristine $\mathrm{mg} / \mathrm{m}^{2}$ & $8.3,1.8-25.9$ & $100 \%$ \\
Doxorubicin $\mathrm{mg} / \mathrm{m}^{2}$ & $53,20-99$ & $15 \%$ \\
Cyclophosphamide $\mathrm{mg} / \mathrm{m}^{2}$ & $1338,556-3375$ & $25 \%$ \\
Etoposide $\mathrm{mg} / \mathrm{m}^{2}$ & $368,218-542$ & $8 \%$ \\
Ifosfamide $\mathrm{mg} / \mathrm{m}^{2}$ & $6824,3034-10,784$ & $7 \%$ \\
Cisplatin $\mathrm{mg} / \mathrm{m}^{2}$ & 111,111 & $1.7 \%$ \\
\hline
\end{tabular}

1991 [4]. This gives us an opportunity to compare the histological findings of the primary biopsy to the nephrectomy sample in order to characterize the effect of the initial chemotherapy.

\section{Methods}

The study cohort was identified by a retrospective review of surgical database for patients operated on for renal tumors in 1988 through 2015 at our hospital. The institutional Ethics Committee had approved the study. Altogether 90 patients were found of whom 70 had had preoperative CNB. We included to the final analysis all the 64 renal tumor patients who had both original preoperative CNB and nephrectomy histology samples available. We excluded the 20 patients who had not undergone preoperative $\mathrm{CNB}$ and six patients whose pathology slides could not be found for re-evaluation.

The general policy of our hospital has been in favor of diagnostic renal biopsy excluding intrarenal stage 1 tumors, cystic tumors and tumors with active bleeding. CNB has been taken by using ultrasound guidance from a posterior or flank approach avoiding the peritoneal cavity. In the early series manually used Tru-Cut ${ }^{\mathrm{TM}}$ cutting needles were used but have been replaced by automatic cutting needles (nowadays: BioPince ${ }^{\circledR}$ 18ga; Argon Medical Devices, TX, USA) during the last 20 years. CNB has been repeated each time until at least two acceptable-quality cylinders were collected.

The pathology slides were re-evaluated by an experienced pediatric pathologist (JL) together with senior pediatric oncologist (MT) to confirm the diagnosis and to classify the response to preoperative chemotherapy according to current guidelines [9]. In case of Wilms' tumor the structured histological evaluation included the estimation of the degree of necrosis (necrotic $=100 \%$ necrosis, regressive $=66-99 \%$ necrosis, poor response $=$ necrosis $<66 \%$ ), proportions of cellular components of Wilms' tumor (epithelial, blastemal, stromal) expressed as a percent of the total surface area of the viable tissue of the tumor, and presence of anaplasia (focal or diffuse) [9].

The complications of the tumor biopsy or nephrectomy as well as the cumulative doses of preoperative chemotherapy agents were collected from the patient charts. Preoperative chemotherapy was based on vincristine and actinomycin D. According to the institutional policy during the early years of the evaluation period, either cyclophosphamide or doxorubicin was used in preoperative chemotherapy of metastatic, anaplastic or blastemal predominance Wilms' tumors (Table 1 ). We

\section{Table 2}

Occurrence of different Wilms's tumor cellular elements from cutting needle biopsy (CNB) at diagnosis and from nephrectomy after preoperative chemotherapy. The figures in parenthesis indicate the frequency of the component in the CNB or nephrectomy sample only.

\begin{tabular}{lllc}
\hline Cellular components & CNB sample & Nephrectomy specimen & p-value \\
\hline Wilms tumor & 59 & 59 & \\
Blastemal cells (n) & $55(17)$ & $40(2)$ & $<0.001$ \\
Epithelial cells (n) & $36(5)$ & $44(13)$ & 0.168 \\
Stromal cells (n) & $45(13)$ & $37(5)$ & $>0.99$ \\
Anaplasia & $3(1)$ & $6^{*}(4)$ & \\
\hline
\end{tabular}

\footnotetext{
* Three patients had diffuse anaplasia and three had focal anaplasia.
}



Fig. 1. Proportion of different cellular elements in 59 Wilms tumor patients. The white and dark boxes indicate the proportion of individual component in the primary cutting needle biopsy and the nephrectomy sample respectively.

evaluated the response to preoperative chemotherapy by analyzing the proportion of tumor necrosis at the time of nephrectomy.

\subsection{Statistical analysis}

Existence of different cellular components in the CNB and in nephrectomy samples were compared by using Fisher's exact test and the change in the proportions of the cellular components with Wilcoxon signed rank test. The association between different cellular components on CNB and the degree of necrosis was evaluated with linear regression. Continuous variables are expressed as medians and interquartile ranges (IQR) or ranges. For the analyses Statview ${ }^{\circledR}$ 5.0.1, SAS Institute Inc. was used. $\mathrm{p}<0.05$ was considered significant.

\section{Results}

\subsection{Patient characteristics}

Twenty-nine of the 64 patients were male. The median age at diagnosis was 3.1 years (IQR 1.8-4.5, range 0.1-17.1). The tumor was located on the right or left side, or bilaterally in 33 (52\%), 27 (42\%) and four (6\%) patients, respectively. Fifteen patients (23\%) presented with lung metastases at diagnosis and five (8\%) had tumor thrombus extending to the vena cava ( 3 with lung metastases) and one patient had a tumor extending to the ureter.

\subsection{Complications of CNB and nephrectomy}

CNB was safe: one patient required a blood transfusion because of bleeding and three patients had hematuria caused by CNB. No other severe side effects were noted. We did not have a tumor rupture during nephrectomy and previous CNB did not complicate nephrectomy and tumor growth was not observed in the CNB tract.

\subsection{Histology in CNB and nephrectomy samples}

Originally 61 tumors got Wilms' tumor diagnosis by CNB. However, after nephrectomy one of them turned out to be clear cell carcinoma and another was primitive neuroectodermal tumor. In the reevaluation, both of these false positive CNBs were found to be insufficient for diagnosis. CNB was considered non-diagnostic both in initial and in re-evaluation in one additional clear cell sarcoma case. The sensitivity of CNB samples both in the original and in this study evaluation 

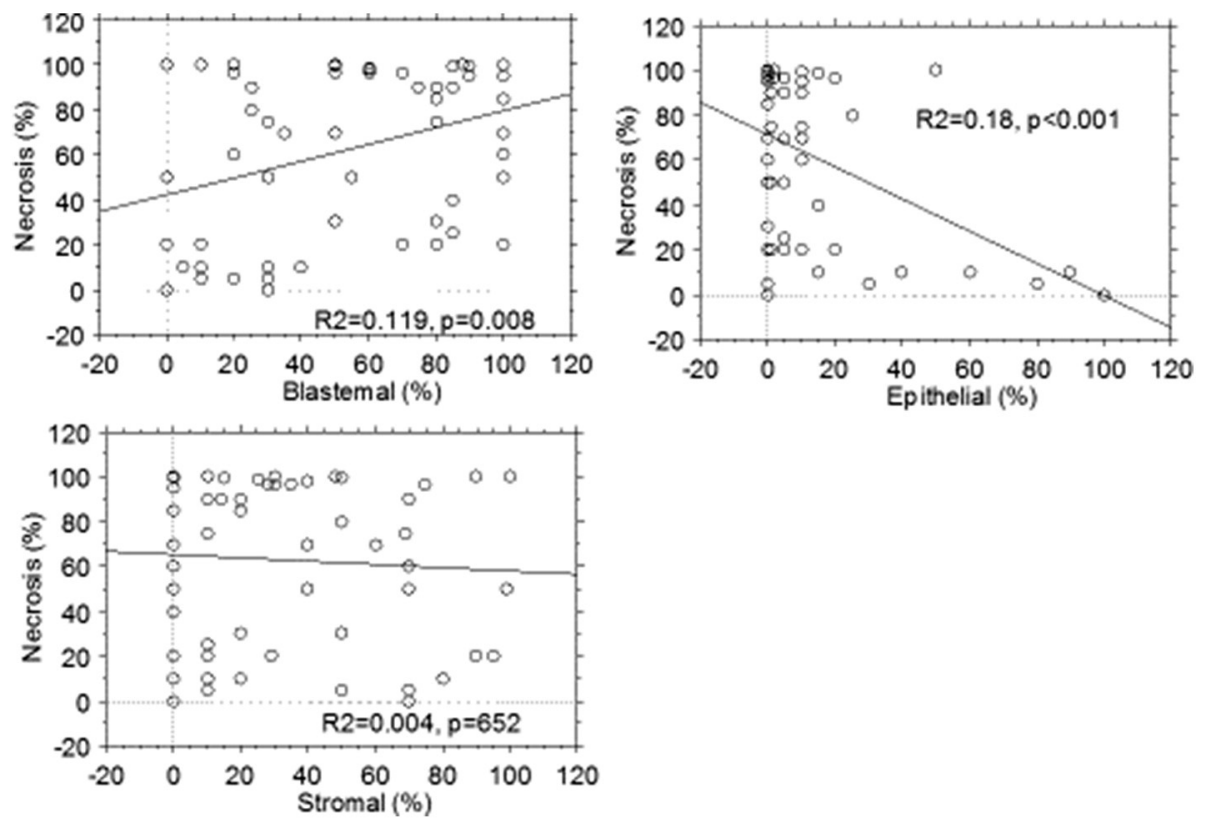

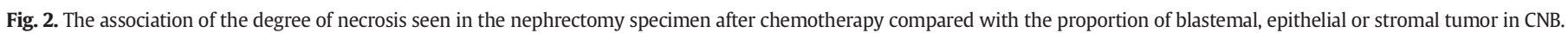

was $100 \%$ for Wilms' tumor cases. In contrast, only two of the five nonWilms' tumors could be diagnosed correctly in CNB samples, these two diagnostic biopsies were a mesoblastic nephroma and the other was a malignant rhabdoid tumor.

In Wilms' tumors blastemal and stromal components were more frequently found in the CNB than in nephrectomy specimens in contrast to the epithelial component (Table 2). Eleven of the diagnostic CBN specimens were solely composed of one histological component that being blastemal in 9 samples and epithelial or stromal in one sample each. Diffuse anaplasia was observed in three nephrectomy samples (5\%). In two of these three cases anaplasia was observed in the diagnostic CNB samples.

The median percentage of blastemal, stromal and epithelial components in the diagnostic CNB in Wilms' tumors was 55\% (IQR 25-85), 28\% (IQR 10-58) and 2\% (IQR 0-10), respectively (Fig. 1). The preoperative chemotherapy resulted in a significant decrease in the proportion of blastemal and an increase in the proportion of epithelial element between the CNB and nephrectomy specimens ( $\mathrm{p}=0.002$ and 0.005 respectively).

The proportion of necrosis seen in the tumors was 80\% (IQR 21-97) in the nephrectomy samples. The nephrectomy specimens were classified as fully necrotic and regressive in $10 \%$ and $44 \%$ respectively [9]. The specimens with least necrosis were of mixed, blastemal, stromal or epithelial type in $20 \%, 7 \%, 13 \%$ and $5 \%$ of cases. A high blastemal cell percentage in the CNB was associated with a high proportion of necrosis and a high epithelial cell content was associated with low proportion of tumor necrosis after preoperative chemotherapy ( $\mathrm{p}=0.008$ and $<0.001$, respectively, Fig. 2).

\subsection{Chemotherapy}

Tumors with high percentage of blastemal component (>50\%) in CNB responded well (median percentage of necrosis $80 \%$ ) to preoperative chemotherapy including vincristine and dactinomycin alone. The addition of alkylating agents or anthracycline did not increase the proportion of necrosis in these blastemal-rich WTs (median percentage of necrosis $97 \%$ ) $(p=0.49)$. The response to VD alone (median percentage of necrosis 50\%) vs. VDA (median percentage of necrosis $20 \%$ ) was similar also among patients with blastemal-poor WTs ( $p=0.88$ ).

\section{Discussion}

CNBs were diagnostic in all Wilms' tumor cases both in initial report and in re-evaluation, given that the sample size was sufficient. However, CNBs were non-diagnostic in three out of the five non- Wilms' cases in re-evaluation. No false negative samples were observed in the reevaluation. Accordingly, no inter-examiner bias was observed in the interpretation of the histological samples of classical Wilms' tumor. The non-Wilms' tumors were more difficult to diagnose in CNB samples and both clear cell sarcoma cases and the primitive neuroectodermal tumor remained undefinable in CNB samples. In classical Wilms' tumors, the high proportion of blastemal cell content in CNB was associated with a large degree of tumor necrosis during pre-operative chemotherapy. In contrast, a high percentage of stromal and especially a high proportion of epithelial component was associated with less tumor necrosis with pre-operative chemotherapy. Consequently, the proportion of blastemal cells decreased and the proportion of epithelial cells increased in response to the pre-operative chemotherapy.

In our study, nephrectomy confirmed Wilms' tumor diagnosis in all 59 cases but only two of the five non-Wilms' tumors were diagnosed by CNB. Similarly, in a study from Sweden all classical Wilms' tumors were found in CNBs, but only one third of non-Wilms' tumors were correctly diagnosed with CNB [7]. As well, anaplasia is challenging to find, both in biopsy and nephrectomy samples. In our study two of the three patients with diffuse anaplasia had signs of anaplasia in CNB samples and similarly in the UK study only 4 out 17 patients with anaplasia were found in CNB [8]. There are also substantial difficulties to find diffuse anaplasia in nephrectomy samples without preoperative chemotherapy; $39 \%$ of the cases detected in central review were not originally found by local pathologist in NWTS study [10].

Blastemal cellular component was the most common component in CNB found in $93 \%$ of the Wilms' tumors in our patients. This is in line with a previous UK study where $89 \%$ of the CNBs of Wilms' tumors patients showed blastemal component [8]. However, blastemal component was less frequent in post-chemotherapy samples, $68 \%$ in our study and $49 \%$ in the UK study. The proportion of stromal element decreased from $76 \%$ to $63 \%$ and the proportion of epithelial element increased from 61 to $75 \%$ in our patients. In the study of Vujanic et al. the decrease was from $66 \%$ to $41 \%$ in stromal elements and from $42 \%$ to $40 \%$ in epithelial elements [8]. The results are also in line with SIOP- 
9/GPOH study in which blastemal predominance was common (39.4\%) in patients with immediate surgery, but rare $(9.3 \%$ ) in patients who had received preoperative chemotherapy whereas regressive changes became common with chemotherapy [11-13]. In SIOP-9 study total necrosis of the tumor was associated with greater volume reduction and better survival [14]. Our study differs from SIOP study by evaluating patients' histopathology before and after chemotherapy in contrast to comparing patients with no chemotherapy versus chemotherapy group, but the message is similar despite in SIOP study the patients were younger and the tumors smaller in in no chemotherapy group [13].

In our study, tumors with a high percentage of blastemal component (> 50\%) responded well to pre-operative chemotherapy consisting of vincristine and dactinomycin alone. The addition of alkylating agents or anthracycline did not increase the proportion of necrosis in the blastemal-rich WTs. This observation from the early series is in line with current practice.

Preoperative chemotherapy may have contributed to safer nephrectomy since we did not have any tumor ruptures during surgery despite of sizeable tumors. This is in accordance to a recent randomized study from UK, in which no per-operative tumor ruptures occurred in patients with preoperative chemotherapy compared to $14.6 \%$ rupture rate in the patients undergoing immediate nephrectomy without chemotherapy [3]. Avoiding tumor rupture and accordingly radiotherapy is of great importance in respect of late effects of cancer therapy in small children.

In our series only one patient needed intervention, transfusion of the red blood cells, because of biopsy. In a previous study, evaluating complications after biopsy $19 \%$ of the patients experienced post-biopsy pain, $20 \%$ had a fall in hemoglobin, $0.5 \%$ needed emergency nephrectomy due to massive bleeding, $0.5 \%$ had tumor rupture perhaps unrelated to the biopsy and $0.5 \%$ got tumor recurrence to the needle track 8 months after the biopsy [8]. Needle tract seeding may be a real risk after percutaneous biopsy $[15,16]$. Thin 18 -gauge needle may decrease the risk of the needle track recurrence after biopsy [7,8]. In our study, viable tumor cells in the CNB tract could not be shown in nephrectomy samples nor were late recurrences detected.

Our study has limitations rising from its retrospective nature. However, an experienced pathologist re-evaluated all the histological samples to cover the possible inter-examiner bias of the histological findings. Because of the heterogeneous nature of the Wilms' tumor, some cellular elements with small percentage are easily missed in CNB decreasing the value of the biopsy. However, our study shows that some cellular elements, especially blastemal may disappear with chemotherapy. Thus, biopsy and nephrectomy samples seem to give supplementary information. Another source of bias may be due to changes in chemotherapy during the study period. We have attempted to control this bias by calculating the cumulative doses of the chemotherapy agents and by evaluating the effect of standard chemotherapy with vincristine and dactinomycin alone or in combination with of alkylating agents or anthracycline both in blastemal-rich and -poor tumors.
We conclude that primary chemotherapy before nephrectomy will result in a median $80 \%$ necrosis of the Wilms' tumor. Best response was seen in blastemal and worst response in epithelial cellular type. However, intensifying preoperative chemotherapy regimen seems not to bring more response in blastemal-rich or blastemal-poor Wilms' tumors, which is in line with current treatment policy. Anaplastic elements and other cellular elements with small volume are easily missed in the diagnostic CNB and non-Wilms' tumors are difficult to recognize. Our study does not support the routine need of core needle biopsy in guidance of pediatric renal tumor pre-operative chemotherapy, but still CNB can be of help in cases with unclear imaging findings if an experienced pathologist is available.

\section{References}

[1] Dome JS, Fernandez CV, Mullen EA, et al. Children's oncology Group's 2013 blueprint for research: renal tumors. Pediatr Blood Cancer 2013;60:994-1000.

[2] Ko EY, Ritchey ML. Current management of Wilms' tumor in children. J Pediatr Urol 2009;5:56-65.

[3] Powis M, Messahel B, Hobson R, et al. Surgical complications after immediate nephrectomy versus preoperative chemotherapy in non-metastatic Wilms' tumour: findings from the 1991-2001 United Kingdom Children's cancer study group UKW3 trial. J Pediatr Surg 2013:48:2181-6.

[4] Saarinen UM, Wikström S, Koskimies O, et al. Percutaneous needle biopsy preceding preoperative chemotherapy in the management of massive renal tumors in children. J Clin Oncol 1991;9:406-15.

[5] Dykes EH, Marwaha RK, Dicks-Mireaux C, et al. Risks and benefits of percutaneous biopsy and primary chemotherapy in advanced Wilms' tumour. J Pediatr Surg $1991 ; 26: 610-2$.

[6] McLorie GA, McKenna PH, Greenberg M, et al. Reduction in tumor burden allowing partial nephrectomy following preoperative chemotherapy in biopsy proved Wilms tumor. J Urol 1991;146:509-13.

[7] Sköldenberg EG, Jakobson A, Elvin A. Pretreatment, ultrasound-guided cutting needle biopsies in childhood renal tumors. Med Pediatr Oncol 1999;32:283-8.

[8] Vujanić GM, Kelsey A, Mitchell C, et al. The role of biopsy in the diagnosis of rena tumors of childhood: results of the UKCCSG Wilmstumor study 3. Med Pediatr Oncol 2003:40:18-22.

[9] Vujanić GM, Sandstedt B. The pathology of Wilms' tumour (nephroblastoma): the International Society of Paediatric Oncology approach. J Clin Pathol 2010;63:102-9.

[10] Dome JS, Cotton CA, Perlman EJ, et al. Treatment of anaplastic histology Wilms tumor: results from the fifth National Wilms' tumor study. J Clin Oncol 2006;24: 2352-8.

[11] Schmidt D, Kramer A, Leuschner I, et al. Tumor regression in nephroblastoma after preoperative chemotherapy according to SIOP 9/GPO: morphological features and relationship to prognosis. Med Pediatr Oncol 1992;20:433.

[12] Weirich A, Schmidt D, Harms D, et al. Distribution of subtypes in standard Wilms tumour after pre-operative chemotherapy and its possible influence on the patients cure rate. Med Pediatr Oncol 1994;23:217.

[13] Weirich A, Leuschner I, Harms D, et al. Clinical impact of histologic subtypes in localized non-anaplastic nephroblastoma treated according to the trial and study SIOP-9 GPOH. Ann Oncol 2001:12:311-9.

[14] Boccon-Gibod L, Rey A, Sandstedt B, et al. Complete necrosis induced by preoperative chemotherapy in Wilms tumor as an indicator of low risk: report of the International Society of Paediatric Oncology (SIOP) Nephroblastoma trial and study 9. Med Pediatr Oncol 2000;34:183-90.

[15] Lee IS, Nguyen S, Shanberg AM. Needle tract seeding after percutaneous biopsy of Wilms tumor. J Urol 1995;153:1074-6.

[16] Aslam A, Foot AB, Spicer RD. Needle track recurrence after biopsy of non-metastatic Wilms tumour. Pediatr Surg Int 1996;11:416-7. 